\title{
Quantum many-body models with cold atoms coupled to photonic crystals
}

\author{
J. S. Douglas ${ }^{1 \star}$, H. Habibian, C.-L. Hung ${ }^{2 \dagger}$, A. V. Gorshkov³ , H. J. Kimble ${ }^{2}$ and D. E. Chang ${ }^{1}$
}

\begin{abstract}
Using cold atoms to simulate strongly interacting quantum systems is an exciting frontier of physics. However, because atoms are nominally neutral point particles, this limits the types of interaction that can be produced. We propose to use the powerful new platform of cold atoms trapped near nanophotonic systems to extend these limits, enabling a novel quantum material in which atomic spin degrees of freedom, motion and photons strongly couple over long distances. In this system, an atom trapped near a photonic crystal seeds a localized, tunable cavity mode around the atomic position. We find that this effective cavity facilitates interactions with other atoms within the cavity length, in a way that can be made robust against realistic imperfections. Finally, we show that such phenomena should be accessible using onedimensional photonic crystal waveguides in which coupling to atoms has already been experimentally demonstrated.
\end{abstract}

7 rapped ultracold atoms are a rich resource for physicists. Isolated from the environment and routinely manipulated, they can act as a quantum simulator for a wide variety of physical models ${ }^{1}$. However, although short-range interactions between atoms can be adjusted by Feshbach resonance, these systems typically lack the long-range interactions required to produce some of the most interesting condensed-matter phenomena. For example, exotic phases such as supersolids are predicted in systems with long-range interactions ${ }^{2}$, as well as Wigner crystallization ${ }^{3}$ and topological states ${ }^{4}$. Long-range interactions can also lead to the breakdown of concepts such as additivity in statistical mechanics ${ }^{5,6}$ and the violation of speed limits (Lieb-Robinson bounds) for the propagation of information ${ }^{7-9}$. As a result, there are active efforts to achieve long-range interactions using specific properties of the atoms ${ }^{10}$, such as their magnetic moment ${ }^{11,12}$, Rydberg excitation ${ }^{13}$ or by using polar molecules ${ }^{14}$.

In this Article we investigate another paradigm, where, instead of relying on atomic properties, we design the medium through which the atoms interact-specifically, by coupling the atoms via the photon modes of a photonic crystal. Our proposal is inspired by demonstrations of strong coupling of photons in nanophotonic systems with individual solid-state emitters ${ }^{15}$ and, more recently, with cold atoms ${ }^{16-19}$. For example, systems of $\sim 10^{3}$ atoms have been trapped by and coupled to the evanescent guided modes of nanofibres ${ }^{16,17}$, and single atoms have been coupled to photonic crystal cavities $^{18}$ and waveguides ${ }^{19}$. One aim of these efforts is to utilize strong, controlled light-matter interactions for quantum information processing and networks ${ }^{20}$. Here, we show that atoms interfaced with photonic crystals can also have remarkable consequences for the exploration of quantum many-body physics ${ }^{21-23}$.

A photonic crystal is a periodic dielectric structure that controls the propagation of light ${ }^{24}$. By introducing a defect into this regular structure, it is possible to induce cavity modes for the light. We demonstrate that a single atom trapped near an otherwise perfect photonic crystal can also seed a localized cavity mode around the atom. The physics of the atom coupled with the photonic crystal can then be understood by a direct mapping to cavity quantum-electrodynamics (QED), allowing intuition and results to be transferred from this well-developed field. When many atoms are trapped, these dynamically induced cavities mediate coherent interactions between atoms ${ }^{25,26}$. The interactions can extend over distances on the order of 100 optical wavelengths and we describe here, for the first time, the principles of how this long-range coupling between atoms can be achieved and tuned in the framework of current experiments.

In particular, going beyond earlier work in this area ${ }^{6,25-30}$, we show how the spatial range and type of effective spin interaction, with the spin encoded in atomic internal degrees of freedom, can be engineered and dynamically tuned using the available atomic structures and by external control of the laser fields. Furthermore, we validate in detail the limits of the applicability of our theoretical model, comparing it with full numerical simulation (Green's function calculations) of an actual one-dimensional photonic crystal waveguide used in experiments. Importantly, we provide realistic descriptions of the fields and atoms, taking into account photon loss and localization of photons due to imperfections in the dielectric structure and loss resulting from free-space atomic emission.

The principles that we elaborate have broad applicability to atom-photon interactions in nanophotonics, including, significantly, two-dimensional photonic crystal geometries ${ }^{31}$. We focus here on implementation in one-dimensional waveguides, and show how such phenomena should be accessible using photonic crystal waveguide geometries in which coupling to atoms has already been experimentally demonstrated ${ }^{19,32}$. More generally, our work provides a platform to realize new regimes of physics involving simultaneous strong and long-range coupling between spins, phonons and photons, enabled by the strong atom-light interactions possible in the nanophotonic system.

\section{Effective long-range interactions}

Long-range interactions between particles often occur through the exchange of photons. A simple example consists of two-level atoms with transition frequency $\omega_{\mathrm{a}}=2 \pi c / \lambda$ interacting via a single mode of a Fabry-Perot cavity that has resonance frequency $\omega_{c}$, as

${ }^{1}$ ICFO-Institut de Ciencies Fotoniques, 08860 Castelldefels, Barcelona, Spain. ${ }^{2}$ Norman Bridge Laboratory of Physics and Institute for Quantum Information and Matter, California Institute of Technology, Pasadena, California 91125, USA. ${ }^{3}$ Joint Quantum Institute and Joint Center for Quantum Information and Computer Science, NIST/University of Maryland, College Park, Maryland 20742, USA. Present address: Department of Physics and Astronomy, Purdue University, West Lafayette, Indiana 47907, USA. ^e-mail: james.douglas@icfo.eu 


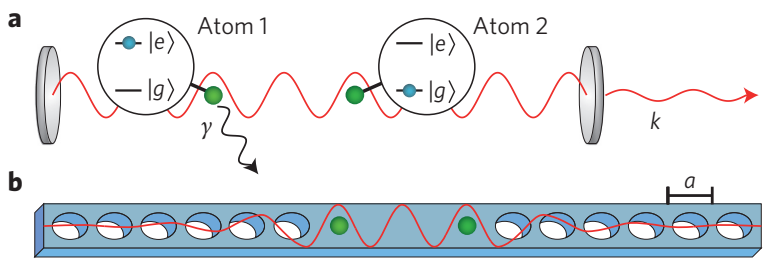

c

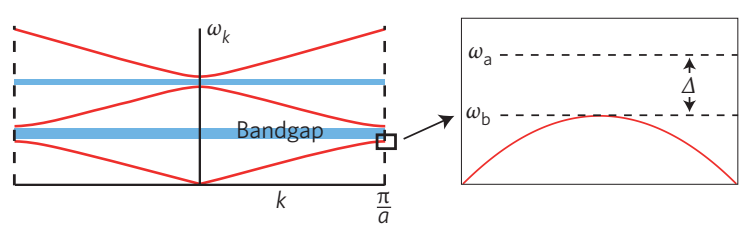

d

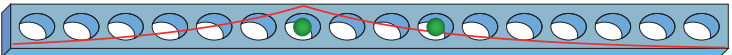

Figure 1 | From cavity-QED to atom-induced cavities in photonic crystals. a, Two atoms are coupled with strength $g_{\mathrm{c}}$ to a single mode of a FabryPerot cavity, enabling an excited atom (atom 1) to transfer its excitation to atom 2 and back. The coherence of this process is reduced by the cavity decay (rate $\kappa$ ) and atomic spontaneous emission into free space (rate $\gamma$ ). b, Photonic crystals are alternating dielectric materials, shown here as oval air holes in a dielectric waveguide, with unit cell length $a$. A defect, such as that caused by removing or altering the hole sizes, can lead to a localized photonic mode (red). Atoms coupled to such a system may then interact via this mode in an analogous manner to that in a. c, A typical band structure of a one-dimensional photonic crystal, illustrating the guided mode frequency $\omega_{k}$ versus the Bloch wavevector $k$ in the first Brillouin zone. We are interested in the case where atoms coupled to the crystal have resonance frequency $\omega_{a}$ close to the band edge frequency $\omega_{b}$, with $\Delta \equiv \omega_{\mathrm{a}}-\omega_{\mathrm{b}}$. d, An atom near a photonic crystal can act as a defect, inducing its own cavity mode with an exponentially decaying envelope (red). A second atom can couple to this mode to produce an interaction similar to that in $\mathbf{a}$ and $\mathbf{b}$, but where the strength now depends on the inter-atomic distance.

shown in Fig. 1a. Momentarily neglecting losses, a single atom in the cavity is described by the Jaynes-Cummings model ${ }^{33}, H=\hbar \omega_{\mathrm{a}} \sigma_{e e}+$ $\hbar \omega_{c} \hat{a}^{\dagger} \hat{a}+\hbar g_{c}\left(\sigma_{e g} \hat{a}+\right.$ h.c. $)$, where $\sigma_{\mu v}=|\mu\rangle\langle v|$ operate on the internal atomic state and the cavity mode excitation has associated annihilation operator $\hat{a}$. The coupling between the atom and the cavity mode $g_{c}=d_{e g} \sqrt{\omega_{c} /\left(2 \hbar \epsilon_{0} V\right)}$ depends on the strength of the dipole matrix element $d_{e g}$ of the two-level transition and on the cavity mode volume $V$. For a single excitation, the eigenstates are dressed states-superpositions of the excitation being purely atomic and purely in the cavity mode-given by $\left|\psi_{1}\right\rangle=\cos \theta|e\rangle|0\rangle+$ $\sin \theta|g\rangle|1\rangle$ and $\left|\psi_{2}\right\rangle=-\sin \theta|e\rangle|0\rangle+\cos \theta|g\rangle|1\rangle$.

When the detuning between the cavity mode and the atomic resonance is large, such that $\Delta_{\mathrm{c}}=\omega_{\mathrm{a}}-\omega_{\mathrm{c}} \gg g_{\mathrm{c}}$, the mixing angle becomes $\theta \approx g_{\mathrm{c}} / \Delta_{\mathrm{c}} \ll 1$, and $\left|\psi_{1}\right\rangle$ is predominantly an atomic excitation with a small photonic component. A second atom introduced into the cavity can then exchange an excitation with the first via the weakly populated cavity mode, leading to an effective interaction. For $N$ atoms this gives ${ }^{33}$

$$
H_{I}=\frac{\hbar g_{\mathrm{c}}^{2}}{\Delta_{\mathrm{c}}} \sum_{j, l}^{N} \sigma_{e g}^{j} \sigma_{g e}^{l}
$$

which describes the exchange of excitations between atoms with a strength that does not diminish with distance, only being bounded by the volume of the physical cavity. These effectively infinite-range interactions, while interesting in their own right ${ }^{34-37}$, remove the spatial complexity of the system, and can often be described using collective operators or mean-field methods.
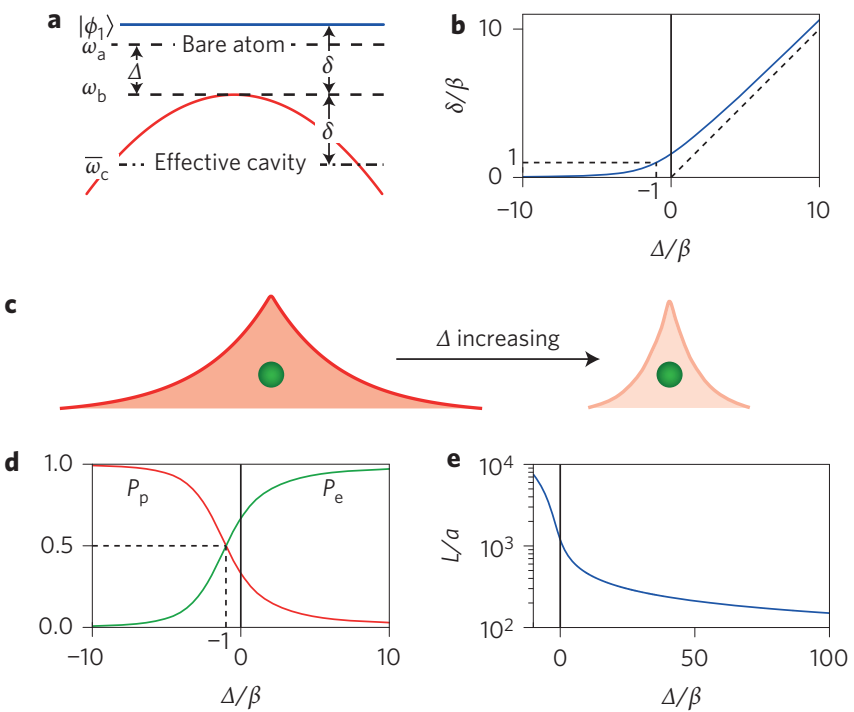

Figure 2 | Effective cavity mode properties. a, Energy level diagram for the photonic crystal dressed state $\left|\phi_{1}\right\rangle$ (blue). The dressed state energy $\omega$ is detuned by $\delta$ from the band edge into the bandgap (band shown in red). The atom is coupled to an effective cavity mode with frequency $\bar{\omega}_{\mathrm{c}}=\omega_{\mathrm{b}}-\delta$ formed by superposition of modes in the band. $\mathbf{b}$, The detuning $\delta$ approaches 0 when $\Delta / \beta \ll-1$ and approaches $\Delta$ when $\Delta / \beta \gg 1$. c, The photonic component of the dressed state has an exponentially decaying envelope around the atomic position. Increasing $\Delta$ decreases the length scale $L$ of the exponential decay and the photonic part of the bound state superposition. d, The atomic excited state population of $\left|\phi_{1}\right\rangle, P_{\mathrm{e}}=\cos ^{2}(\theta)$ (green), increases as a function of $\Delta$, while the population of the photon mode, $P_{\mathrm{p}}=\sin ^{2}(\theta)$ (red), decreases as the state switches from photonic to atomic. $\mathbf{e}$, The length of the effective cavity decreases with $\Delta$. Here $L$ is in units of the lattice constant $a$, calculated for $\alpha=10.6$ and $\beta=4.75 \times 10^{-7} \omega_{\mathrm{b}}$, which is consistent with the 'alligator' photonic crystal waveguide (see main text) ${ }^{32}$.

To realize long-range interactions that decay with distance we utilize photonic crystals. Key to our proposal is that through constructive interference of light scattering from the crystal's periodic structure, frequency windows known as bandgaps can be created in which no propagating modes exist. Figure 1c shows a typical dispersion relation of photon frequency $\omega_{k}$ versus Bloch wavevector $k$ with a bandgap. Conventionally, a localized photonic crystal cavity mode is created by introducing a local dielectric defect (Fig. 1b) that pulls a discrete mode into the bandgap from the continuous band spectrum $^{24}$. Here, we show that an atom trapped near the photonic crystal is itself a dielectric defect capable of seeding a cavity mode localized around the atomic position, via which it can interact with other atoms (Fig. 1d).

\section{Atom-induced cavities and long-range interactions}

The interaction between atoms and band edges has been discussed in a number of contexts, such as the formation of atom-photon bound states ${ }^{27}$, radiative coupling between atoms $s^{25,26,28}$ and spinentanglement ${ }^{30}$ and thermalization ${ }^{6}$ mediated by long-range interactions. Here, we provide an elegant interpretation of this physics in terms of atom-induced cavities and cavity QED. This mapping enables the powerful toolbox of cavity QED to be transferred to these systems and enables one to identify key figures of merit (such as mode volume and cooperativity parameter). We exploit this mapping to demonstrate that the type of spin interaction and the spatial range can be manipulated dynamically, enabling tunable access to a wide range of long-range interacting models. We also identify the limits imposed by system imperfections 
(such as losses and disorder), and analyse in detail a realistic structure wherein this novel long-range physics can be realized.

A simple model to illustrate this mechanism consists of two-level atoms coupled to the photonic crystal modes, where the atomic resonance is close to one of the band edges of the photonic crystal (Fig. 1c) with detuning $\Delta=\omega_{\mathrm{a}}-\omega_{\mathrm{b}}$. We assume that the detuning to any other band edge is much larger than $\Delta$, so the influence of other bands is negligible. When the atomic resonance is close to the band edge, the atom is dominantly coupled to modes close to the band edge wavevector $k_{0}$ due to the van Hove singularity in the density of states. In this case we can approximate the dispersion relation to be quadratic $\omega_{k} \approx \omega_{\mathrm{b}}\left(1-\alpha\left(k-k_{0}\right)^{2} / k_{0}^{2}\right)$ about $k_{0}$, where $\alpha$ characterizes the band curvature ${ }^{27}$. Due to the periodicity of the photonic crystal the photonic modes are of Bloch form and the modes with wavevector $k \sim k_{0}$ take the form $E_{k}(z) \approx e^{i k z} u_{k_{0}}(z)$, with annihilation operator $\hat{a}_{k}$. Furthermore, for these modes, the coupling $g=d_{e g} \sqrt{\omega_{b} /\left(4 \pi \hbar \varepsilon_{0} A\right)}$ is approximately independent of $k$, where $A$ is the mode cross-sectional area ${ }^{38}$.

A system with one atom trapped at $z=0$ coupled to the photonic crystal is then described by the Hamiltonian

$$
H=\hbar \omega_{\mathrm{a}} \sigma_{e e}+\int \mathrm{d} k \hbar \omega_{k} \hat{a}_{k}^{\dagger} \hat{a}_{k}+\hbar g \int \mathrm{d} k\left(\sigma_{e g} \hat{a}_{k} E_{k}(0)+\text { h.c. }\right)
$$

For a single excitation in the system, solving the Schrödinger equation, $H|\psi\rangle=\hbar \omega|\psi\rangle$, yields the dressed state $\left|\phi_{1}\right\rangle=\cos \theta|e\rangle|0\rangle+$ $\sin \theta|g\rangle|1\rangle$, where the atom is dressed by a localized photonic mode $|1\rangle=\int \mathrm{d} k c_{k} \hat{a}_{k}^{\dagger}|0\rangle$ (Supplementary Section 1). The eigenfrequency $\omega$ lies within the bandgap, with detuning $\delta=\omega-\omega_{\mathrm{b}}>0$ from the band edge, as shown in Fig. 2a,b. The detuning $\delta$ is the positive real root of $(\delta-\Delta) \sqrt{\delta}=2 \beta^{3 / 2}$ for $\beta=\left(\pi g^{2}\left|u_{k_{0}}(0)\right|^{2} k_{0} / \sqrt{4 \alpha \omega_{\mathrm{b}}}\right)^{2 / 3}$.

Importantly, the photonic component is localized around the atomic position, as illustrated in Fig. 2c, with spatial mode function

$$
\phi(z)=\int \mathrm{d} k c_{k}^{*} E_{k}(z)=\sqrt{\frac{2 \pi}{L}} e^{-|z| / L} E_{k_{0}}(z)
$$

The photon decays exponentially with distance $z$ from the atomic position with length scale $L=\sqrt{\alpha \omega_{\mathrm{b}} /\left(k_{0}^{2} \delta\right)}$, reflecting the fact that within the bandgap at energy $\omega$, the field propagation equation has complex solutions with attenuation length $L$.

This confined photonic cloud has the same properties as a real cavity mode, enabling a mapping onto the Jaynes-Cummings model. Specifically, one can associate an effective atom-cavity interaction strength $\bar{g}_{\mathrm{c}}=g \sqrt{2 \pi / L}$ with the bandgap system, which depends on the mode volume, expressed here as the effective cavity length given by the decay length $L$ for fixed mode area $A$. We can also identify an effective cavity mode frequency that is the average frequency of the photon mode, $\int \mathrm{d} k\left|c_{k}\right|^{2} \omega_{k}=\omega_{\mathrm{b}}-\delta$. The effective atom-cavity detuning is $\bar{\Delta}_{\mathrm{c}}=\Delta+\delta$, as shown in Fig. $2 \mathrm{a}$ (note that for $\Delta=-\beta$ the effective cavity is resonant with the atom $\left.\bar{\Delta}_{c}=0\right)$. The state $\left|\phi_{1}\right\rangle$ then maps to the dressed state $\left|\psi_{1}\right\rangle$ from the Jaynes-Cummings model; that is, the mixing angle and energy are the same for $\bar{\Delta}_{\mathrm{c}} \rightarrow \Delta_{\mathrm{c}}$ and $\bar{g}_{\mathrm{c}} \rightarrow g_{\mathrm{c}}$. This mapping breaks down when we consider the second dressed state, which is not an eigenstate in the photonic crystal model because of the continuum of propagating modes for frequencies below $\omega_{\mathrm{b}}$.

The atomic excited-state population $P_{\mathrm{e}}=\cos ^{2} \theta$ for $\left|\phi_{1}\right\rangle$ is plotted in Fig. 2 d. For $\Delta \gg \beta$, most of the excitation resides in the atom, while for $\Delta \ll-\beta$ the state becomes mostly photonic, a cavity mode dressed by the atom. Physically, although the atomic frequency can lie well within the band, the atom still provides a weak refractive index contrast at frequencies within the gap, and in one dimension, arbitrarily weak dielectric defects can seed a cavity mode ${ }^{39}$. In this regime (Fig. 2e), the weak dielectric contrast yields a very long effective cavity length. In practice, this length is only limited by the finite size of the photonic crystal structure or by disorder in the photonic crystal structure (as discussed below).

It is now apparent that two atoms can exchange an excitation via the induced cavity mode provided they are separated by a distance on the order of the decay length $L$. In the limit where the photonic modes are weakly populated $(\Delta \gg \beta)$, this leads to an effective dipole-dipole interaction between atoms, with positions $z_{j}$, of the form (Supplementary Section 2)

$$
H_{I} \approx \frac{\hbar \bar{g}_{\mathrm{c}}^{2}}{\bar{\Delta}_{\mathrm{c}}} \sum_{j, l}^{N} \sigma_{e g}^{j} \sigma_{g e}^{l} f\left(z_{j}, z_{l}\right)
$$

The effective atom-cavity detuning is $\bar{\Delta}_{\mathrm{c}}=2 \Delta$ (since $\delta \sim \Delta$ ) and $f\left(z_{j}, z_{l}\right)=e^{-\left|z_{j}-z_{l}\right| / L} E_{k_{0}}\left(z_{j}\right) E_{k_{0}}^{*}\left(z_{l}\right)$ (ref. 30).

Compared to the case of a conventional cavity (equation (1)), the main feature of interactions emerging from atom-induced cavities is that the spatial function $f\left(z_{j}, z_{l}\right)$ is finite in range and tunable, both through the effective interaction length $L$ and the Bloch functions (for example, from which changes in sign can be engineered). In addition, this dynamic cavity mode follows the atomic position rather than being a static property set by boundary conditions. Note that although exponentially decaying interactions are identified as short-range in the thermodynamic limit, the length scale $L$ can be on the order of the length of experimental systems, and thus effectively long-range over the system size ${ }^{6,30}$. Furthermore, as we show below, the interaction can approximate long-range power laws over a finite system, similar to the case in trapped ion experiments $8,9,40,41$. These results also generalize to higher dimensions (Supplementary Section 2). For example, in a two-dimensional photonic crystal atom-induced cavities lead to the function $f\left(\mathbf{z}_{j}, \mathbf{z}_{l}\right) \approx E_{\mathbf{k}_{0}}\left(\mathbf{z}_{j}\right) E_{\mathbf{k}_{0}}^{*}\left(\mathbf{z}_{l}\right) e^{-\left|\mathbf{z}_{j}-\mathbf{z}_{l}\right| / L} / \sqrt{\left|\mathbf{z}_{j}-\mathbf{z}_{l}\right|}$ (ref. 31).

Although equation (4) superficially looks like a long-range spin model, the possible dynamics are in fact much richer. In particular, treating the atomic positions themselves as dynamical variables, the function $f\left(z_{j}, z_{l}\right)$ can be interpreted physically as a mechanical potential acting on atoms $s^{6}$, which is turning on and off as spin degrees of freedom change. Due to the large values of the effective vacuum Rabi splittings $\bar{g}_{c}$ associated with the dynamic atomic cavities, the strength of these spin-dependent potentials can be extremely large compared to typical motional energy scales associated with ultracold atoms. Furthermore, in the nanophotonic system, it is possible to achieve strong spin-photon coupling (for example, an incident photon can be absorbed by the atoms with high probability ${ }^{19}$ ). Thus, our system produces a unique coupling over a long range between spin, phonon and photon degrees of freedom.

\section{Coherence and effective cooperativity}

Key to any physical realization of the long-range physics we describe above is how dissipation competes with the coherent interaction in equation (4). Here, we go beyond previous discussions of this type of interaction by detailing the limits imposed by realistic loss mechanisms and experimental imperfections and further show these interactions should be observable in current state-of-the-art experiments.

Imperfections in the photonic crystal cause photon loss at rate $\kappa_{\mathrm{p}}$, and because our structures of interest are not full three-dimensional photonic crystals, an excited atom can spontaneously emit into free space at rate $\gamma$ (usually comparable to the vacuum rate $\gamma_{0}$, ref. 38). The effect of losses can be revealed, for example, by studying the exchange of an excitation between two atoms separated by $\left|z_{1}-z_{2}\right| \lesssim L$. From equation (4), the exchange time is given by $\tau \sim \pi \bar{\Delta}_{\mathrm{c}} /\left(2 \bar{g}_{\mathrm{c}}^{2}\right)$, while the total loss is given by $\tau\left(\gamma \cos ^{2} \theta+\kappa_{\mathrm{p}} \sin ^{2} \theta\right)$. Optimizing the detuning, we find an exchange error of $\pi / \sqrt{C}$, where $C=\bar{g}_{c}^{2} /\left(\kappa_{\mathrm{p}} \gamma\right)$ is the single-atom cooperativity (see Methods). For a stateof-the-art photonic crystal $(Q \approx 200,000)$ coupled to caesium atoms $(\gamma /(2 \pi) \approx 5 \mathrm{MHz})$, a cavity with volume $V \approx \lambda^{3}$ (that is, $\left.L=\lambda\right)$ could 
have $\bar{g}_{c} /(2 \pi) \approx 10 \mathrm{GHz}$, giving a feasible cooperativity of $C_{\lambda} \approx 10^{4}$ (ref. 32). Assuming that $\kappa_{\mathrm{p}}$ is dominated by local imperfections in the photonic crystal, we expect that it is independent of length $L$ (as compared to a Fabry-Perot cavity in vacuum, where $\kappa_{\mathrm{p}} \propto 1 / L$ ). The cooperativity of the dynamic cavity mode then scales with length as $C_{L}=\lambda C_{\lambda} / L$, which limits the length for which interactions remain coherent. For $C_{\lambda} \approx 10^{4}, C_{L}$ remains greater than 100 for lengths of up to 100 wavelengths.

Beyond the photon losses already discussed, imperfections in photonic crystal fabrication can yield disordered potentials for propagating fields. Disorder results in an Anderson localization length over which optical fields tend to become trapped, limiting the interaction range between atoms. As discussed in Supplementary Section 4 , the physics of weak disorder near a band edge leads to a universal scaling for the localization length as a function of the level of disorder. This scaling predicts that localization lengths exceeding 100 wavelengths are possible with state-of-the-art fabrication and is consistent with separate (unpublished) experimental characterization of the 'alligator' structure from ref. 32, which reveals a localization length longer than the structure length of $\sim 200$ unit cells. On the other hand, disorder may prove to be a feature of the system, providing access to physical models with interactions that have randomly varying length scales.

Implementation in an 'alligator' photonic crystal waveguide Our simple theoretical model should also approximate actual physical implementations. As a concrete example, we consider the onedimensional 'alligator' photonic crystal waveguide (APCW) as experimentally demonstrated in refs 19 and 32. The APCW, as shown in Fig. 3a, consists of two parallel, periodically corrugated nanobeams, whose small distance of separation couples and hybridizes their optical modes. A combination of far off-resonant guided modes and Casimir-Polder forces allows atoms to be localized between the beams at the periodic points indicated in red in Fig. $3 \mathrm{a}^{38}$.

The band structure of the APCW, calculated using the MIT Photonic-Bands software package, is shown in Fig. 3a. The band edge of the fundamental transverse electric (TE-like) mode, located at $\omega_{\mathrm{b}} / 2 \pi=333 \mathrm{THz}$, is closely aligned with the $D_{1}$ transition of atomic caesium to produce the desired long-range interactions. In Fig. 3b, we plot the coefficients $\left|U_{j l}\right| / \gamma_{0}$ for the effective atom-atom interaction $H_{I}=\hbar \sum_{j, l} U_{j l} \sigma_{e g}^{j} \sigma_{g e}^{l}$ calculated numerically for the full structure of the APCW (see Methods). Couplings are plotted for detunings of the atom from the band edge ranging from $\Delta / 2 \pi=400 \mathrm{GHz}$ to $2.8 \mathrm{THz}$, over atomic separations $r_{j l} / a$ extending up to 55 lattice sites $(a=371 \mathrm{~nm}$ is the lattice constant of the APCW). The predictions from our simple model, equation (4) (solid lines in Fig. 3b), quantitatively agree with the numerical simulation for structure band curvature $\alpha=10.6$ and coupling $\bar{g}_{c} / 2 \pi=\sqrt{a / L} \times 12.2 \mathrm{GHz}$ (see Methods). The deviation between the theoretical and numerical results at $\Delta / 2 \pi=400 \mathrm{GHz}$ is primarily attributable to finite-size effects, as the interaction length becomes comparable to the simulated structure size ( $75 a$ between the source and either end of the APCW).

At short atomic separations $r_{j l} / a \lesssim 15$, numerical results deviate from the model at all detunings (Fig. 3b). We primarily attribute this difference to the contributions coming from other guided bands (see ref. 19 for the full band structure), as well as leaky and free-space modes that are not included in our single-band theoretical model. The band edges of these modes are typically far from the atomic transition frequency, leading to contributions of the order of the interaction strength between atoms coupled via a nanofibre or free space, that is, at most the free-space linewidth $\gamma_{0}$ (refs 42,17). The fractional error associated with these corrections will then become smaller as experimental systems are optimized to increase the interaction strength arising from the primary mode. This can be done by working closer to the band edge (seen only to a
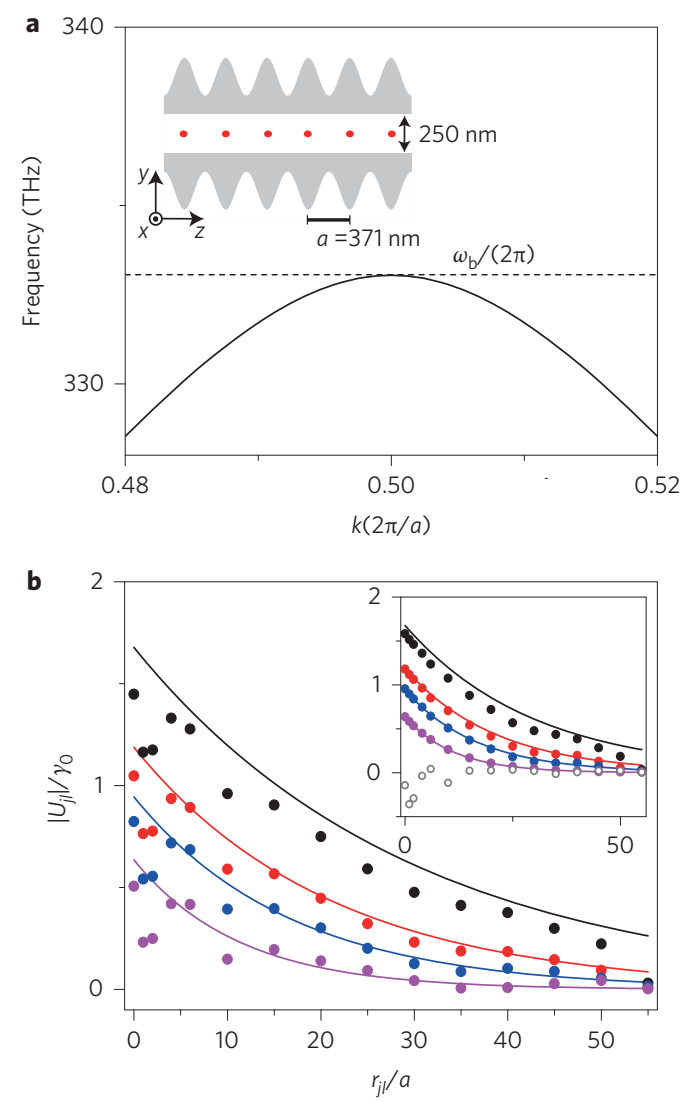

Figure 3 | Comparison of the single-band model with numerical calculations. a, Band structure of the fundamental TE mode of the onedimensional 'alligator' photonic crystal waveguide (APCW), designed for coupling to the $D_{1}$ line of atomic caesium near the photonic band-edge frequency $\omega_{\mathrm{b}} / 2 \pi=333 \mathrm{THz}$ (refs 19,32). The calculated band structure has a curvature $\alpha \approx 10.6$ near the band edge at $k_{0}=\pi / a$. Inset: the dielectric profile of the APCW. Red circles denote the location of trapped atoms. b, Atom-atom coupling strength $U_{i j}$ evaluated using FDTD simulations (solid circles) and the single-band model from equation (4) (solid lines). Results are plotted for atomic detunings from the band edge $\Delta / 2 \pi=400$ (black), 800 (red), 1,300 (blue), 2,800 (magenta) GHz. Inset: FDTD results where the contribution from all other photonic and free-space modes in the APCW (open circles) has been estimated numerically and subtracted (see Methods).

limited extent in Fig. $3 b$ because of finite size effects), or by using structures with smaller band curvature $\alpha$ to decrease the interaction length. Even better agreement with the model can be reached by approximately subtracting out the contributions from the other modes (see Methods), as plotted in the inset of Fig. 3b, supporting the validity of our simple model.

\section{Designing interaction properties}

The long-range interactions given in equation (4) depend on the detuning from the band edge $\Delta$ and band curvature $\alpha$, which cannot be easily tuned given a physical structure. This is remedied by considering atoms with an internal $\Lambda$-level structure, as shown in Fig. $4 \mathrm{a}$, introducing an additional metastable state $|s\rangle$. Here the $|g\rangle-|e\rangle$ transition is coupled to the band edge as before, while $|s\rangle-|e\rangle$ is assumed to be de-coupled but addressable by an external laser (for example, illuminating the photonic crystal from the side) with Rabi amplitude $\Omega$ and detuning $\delta_{L}$. This situation may be achieved, for example, if the photonic crystal modes and external laser have orthogonal polarizations. Alternatively, guided modes of the photonic crystal with orthogonal polarization may be used. 

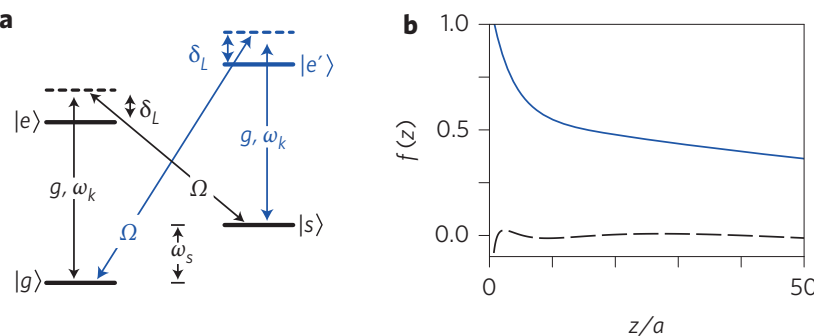

Figure 4 | Designing interaction potentials. a, Driven (black) $\Lambda$ and (black and blue) four-level system. In the $\Lambda$ scheme, transition $|g\rangle-|e\rangle$ couples with strength $g$ to the photonic crystal modes, while $|s\rangle-|e\rangle$ is pumped by a laser with detuning $\delta$ and Rabi frequency $\Omega$. Interactions between the $x$-component of the effective spin can be achieved by adding level $\left|e^{\prime}\right\rangle$, where the transition $|s\rangle-\left|e^{\prime}\right\rangle$ also couples to the modes of the photonic crystal, while a second pump drives $|g\rangle-\left|e^{\prime}\right\rangle$. b. Approximate power law interactions between atoms over a finite region can be achieved by summing the different exponential interactions associated with multiple drive fields. This is illustrated here over 50 lattice sites, where two exponentials are added to yield an $\eta=1 / 4$ power law: $f(z)=w_{1} e^{-s_{1} z / a}+w_{2} e^{-s_{2} z / a} \approx z^{-1 / 4}$ (solid blue curve). Error $f(z)-z^{-1 / 4}$ is given by the dashed curve. Here $w_{1}=0.5480, w_{2}=0.5684, s_{1}=0.2916$ and $s_{2}=0.0089$ could be achieved by detuning one laser from the band edge by $1.723 \times 10^{-3} \omega_{\mathrm{b}}$ and the second by $1.612 \times 10^{-6} \omega_{\mathrm{b}}$ for $\alpha=0.2$

The photons in the system are now Raman scattered from the laser field with central frequency $\omega_{\mathrm{a}}+\delta_{L}$ and intuitively the photon cloud size will be determined by the attenuation length at this frequency rather than at $\omega_{\mathrm{a}}$ as in the case of pure atomic excitation. Specifically, by adiabatically eliminating the excited state and the photonic modes (Supplementary Section 2) we obtain an interaction within the ground-state manifold given by

$$
H_{I}=\frac{\hbar|\Omega|^{2} \bar{g}_{\mathrm{c}}^{2}}{2 \Delta_{L} \delta_{L}^{2}} \sum_{j, l}^{N} \sigma_{s g}^{j} \sigma_{g s}^{l} f\left(z_{j}, z_{l}\right)
$$

This interaction is of the same form as in equation (4) where $\Delta=\omega_{\mathrm{a}}-\omega_{\mathrm{b}}$ is now replaced by $\Delta_{L}=\delta_{L}+\omega_{\mathrm{a}}-\omega_{\mathrm{b}}$ and $\bar{g}_{\mathrm{c}}$ is replaced by $\Omega \bar{g}_{c} / \delta_{L}$. The strength of the interaction is reduced by a factor of $|\Omega|^{2} / \delta_{L}^{2}$, leading to an increase in the time needed for the spin exchange; however, the population of the atomic excited state is also reduced by the same factor reducing the rate of spontaneous emission. The Raman process effectively narrows the natural line width of the excited state, and the cooperativity $C$, which characterizes the optimal fidelity of exchange, remains constant.

By tuning the frequency and amplitude of the drive laser, the interaction strength and length $L=\sqrt{\alpha \omega_{\mathrm{b}} /\left(\Delta_{L} k_{0}^{2}\right)}$ can now be dynamically altered. It also becomes feasible to build interaction scalings other than exponential, by driving the $|s\rangle-|e\rangle$ transition with two or more fields of different frequencies. This leads to an interaction potential for the atoms that is the sum of the potentials due to each individual drive field (the adiabatic elimination of drive fields is additive). For example, a power law interaction $f\left(z_{j}, z_{l}\right) \propto\left|z_{j}-z_{l}\right|^{-\eta}$ can be approximated over a finite range, as we show in Fig. $4 \mathrm{~b}$ for $\eta=1 / 4$ over 50 unit cells using two pump fields.

The full spin-phonon-photon dynamics resulting from the coupling of atoms to the photonic crystal bands enables a wide range of behaviour to be investigated. In addition, the limiting cases of the model, found by freezing out degrees of freedom, are in themselves interesting. For example, the motional modes may be eliminated by trapping atoms tightly on a lattice to produce quantum magnetism models, such as the XX-model and the transverse Ising model (see Methods), where long-range interactions lead to a breakdown of Lieb-Robinson bounds ${ }^{7-9}$. Alternatively, in the opposite limiting case, we may focus on the motional dynamics of the atoms, eliminating the spin dynamics by driving the atoms weakly and off resonance. In this case the interaction yields a purely mechanical potential for the atoms, $U \approx\left(\hbar|\Omega|^{2} g_{c}^{2} / 2\left(\omega_{L}-\omega_{\mathrm{b}}\right)\left(\omega_{L}-\omega_{\mathrm{a}}\right)^{2}\right) f\left(z_{j}, z_{l}\right)$. Engineering this interaction to be a power law with $\eta=1$, for example, would enable simulation of charged particles using neutral cold atoms.

\section{Discussion}

In conclusion, we have demonstrated the utility of atoms coupled to photonic crystal structures as a toolbox to achieve tunable longrange interactions in many-body systems. Moreover, significant progress has been made to experimentally realize systems in which the predicted rich interplay between spin, motional and photonic degrees of freedom could be observed. Individual atoms have been trapped and coupled to a photonic crystal cavity using optical tweezers $^{18}$ and atoms may also be trapped in the evanescent field of guided modes of photonic crystals ${ }^{19,32,38}$.

In these systems, many-body phenomena such as frustration ${ }^{43}$, information propagation ${ }^{7-9}$ and many-body localization ${ }^{44}$ may be investigated in the presence of long-range interactions. Such interactions could also generate nonlocal nonlinearities for photons, leading to photonic molecules and other exotic states ${ }^{45,46}$. Finally, the use of band edges to manipulate interactions should find wide use beyond the photonic crystal setting. In the context of atomphoton interactions, band structure could be engineered using a variety of fabrication techniques ${ }^{32,47}$, or using periodic arrangements of atoms themselves ${ }^{48}$, while coupling of quantum bits via phononic bands ${ }^{49}$ could be controlled in an analogous manner.

\section{Methods}

Coherence and effective cooperativity. The optimal loss rate connected with the cooperativity parameter is reached by adjusting the detuning of the effective cavity. However, adjusting only the detuning also changes the length scale. To keep the

length $L=\sqrt{\alpha \omega_{\mathrm{b}} /\left(\Delta k_{0}^{2}\right)}$ fixed and achieve the optimal detuning we must also adjust the band curvature $\alpha$. The APCW photonic crystal structures to which atoms have already been successfully coupled have curvature parameters on the order of $\alpha \approx 10$, giving an optimal length of $L \approx 100 \lambda$ with $C_{L} \approx 100$ for the parameters given in the main text. Slight design changes should enable values of $\alpha \approx 1$ leading to optimal length $L \approx 20 \lambda$ and $C_{L} \approx 600$ (ref. 38). Reaching shorter interaction lengths with a fidelity limited by $C_{L}$ requires structures with even flatter bands, and the design of such systems that are also compatible with atom trapping is being investigated further.

Implementation in an 'alligator' photonic crystal waveguide. Couplings $U_{j l}$ are calculated assuming the atoms are at the minima of the APCW trapping potentials and the atomic transition is polarized along $\hat{y}$ (the local direction of polarization for the fundamental TE mode). The $U_{j l}$ are directly proportional to the real part of the dyadic electromagnetic The Green's function $G_{y y}\left(r_{j}, r_{l}, \omega_{\mathrm{a}}\right)^{50}$. The Green's function physically describes the field produced at position $r_{j}$ due to a source at $r_{l}$ and frequency $\omega_{\mathrm{a}}$ and is obtained for the APCW by finite-difference time-domain (FDTD) simulations. The coupling coefficients normalized by the free-space emission rate are $\left|U_{j l}\right| / \gamma_{0}=\left|\operatorname{Re} G_{y y}\left(r_{j}, r_{l}, \omega_{a}\right)\right| /\left(2 \operatorname{Im} G_{y y}\right.$,free $\left.\left(0,0, \omega_{\mathrm{a}}\right)\right)$, where $G_{\text {free }}$ is the free-space Green's function. To check the validity of our theoretical model given by equation (4), we use a parameter of $\alpha=10.6$ describing the actual curvature near the band edge for the APCW ${ }^{32}$. The atom-field coupling strength $\bar{g}_{c}$ is obtained from first principles by numerical quantization of the guided modes near the band edge (Supplementary Section 3), yielding $\bar{g}_{c} / 2 \pi=\sqrt{a / L} \times 12.2 \mathrm{GHz}$.

To estimate the contribution to the interaction coefficient from other modes, we note that leaky and free-space modes should exhibit negligible frequency dependence over the narrow range of detunings $\Delta$ considered. We then estimate the multimode contribution by calculating the difference between the model and numerical $\left|U_{j l} / \gamma_{0}\right|$ at $\Delta / 2 \pi=3 \mathrm{THz}$

Designing interaction properties. The interaction in equation (5) correspond to the XX-model, $\sum\left(\sigma_{x}^{j} \sigma_{x}^{l}+\sigma_{y}^{j} \sigma_{y}^{l}\right) f\left(z_{j}, z_{l}\right)$, for spin-1/2 operators $\left(\sigma_{x}, \sigma_{y}, \sigma_{z}\right)=\left(\sigma_{g s}+\sigma_{s g}^{j \neq} l i\left(\sigma_{g s}-\sigma_{s g}\right), \sigma_{s s}-\sigma_{g g}\right) / 2$. Other level schemes, such as the four-level structure in Fig. $4 \mathrm{a}$, enable other spin models. Here, the $\Lambda$-structure is extended by adding a transition $|s\rangle-\left|e^{\prime}\right\rangle$ that also couples to the photonic crystal modes, while a second pump with the same amplitude and detuning as the first drives the $|g\rangle-\left|e^{\prime}\right\rangle$ transition. Eliminating the excited-state manifold 
(Supplementary Section 2) now yields an effective Hamiltonian corresponding to the transverse Ising model with long-range interactions,

$$
H=\hbar \omega_{s} \sum_{j}^{N} \sigma_{z}^{j}+\frac{2 \hbar|\Omega|^{2} \bar{g}_{c}^{2}}{\Delta_{L} \delta_{L}^{2}} \sum_{j \neq l}^{N} \sigma_{x}^{j} \sigma_{x}^{l} f\left(z_{j}, z_{l}\right)
$$

Received 18 October 2014; accepted 5 March 2015; published online 6 April 2015

\section{References}

1. Bloch, I., Dalibard, J. \& Nascimbene, S. Quantum simulations with ultracold quantum gases. Nature Phys. 8, 267-276 (2012).

2. Batrouni, G. G., Scalettar, R. T., Zimanyi, G. T. \& Kampf, A. P. Supersolids in the Bose-Hubbard Hamiltonian. Phys. Rev. Lett. 74, 2527-2530 (1995).

3. Wigner, E. On the interaction of electrons in metals. Phys. Rev. 46, 1002-1011 (1934).

4. Micheli, A., Brennen, G. K. \& Zoller, P. A toolbox for lattice-spin models with polar molecules. Nature Phys. 2, 341-347 (2006).

5. Campa, A., Dauxois, T. \& Ruffo, S. Statistical mechanics and dynamics of solvable models with long-range interactions. Phys. Rep. 480, 57-159 (2009).

6. Shahmoon, E., Mazets, I. \& Kurizki, G. Non-additivity in laser-illuminated many-atom systems. Opt. Lett. 39, 3674-3677 (2014).

7. Hauke, P. \& Tagliacozzo, L. Spread of correlations in long-range interacting quantum systems. Phys. Rev. Lett. 111, 207202 (2013).

8. Richerme, P. et al. Non-local propagation of correlations in quantum systems with long-range interactions. Nature 511, 198-201 (2014).

9. Jurcevic, P. et al. Quasiparticle engineering and entanglement propagation in a quantum many-body system. Nature 511, 202-205 (2014).

10. Lahaye, T., Menotti, C., Santos, L., Lewenstein, M. \& Pfau, T. The physics of dipolar bosonic quantum gases. Rep. Prog. Phys. 72, 126401 (2009).

11. Griesmaier, A., Werner, J., Hensler, S., Stuhler, J. \& Pfau, T. Bose-Einstein condensation of chromium. Phys. Rev. Lett. 94, 160401 (2005).

12. Lu, M., Burdick, N. Q., Youn, S. H. \& Lev, B. L. Strongly dipolar Bose-Einstein condensate of dysprosium. Phys. Rev. Lett. 107, 190401 (2011).

13. Saffman, M., Walker, T. G. \& Mølmer, K. Quantum information with Rydberg atoms. Rev. Mod. Phys. 82, 2313-2363 (2010).

14. Ni, K.-K. et al. A high phase-space-density gas of polar molecules. Science 322, 231-235 (2008).

15. Khitrova, G., Gibbs, H. M., Kira, M., Koch, S. W. \& Scherer, A. Vacuum Rabi splitting in semiconductors. Nature Phys. 2, 81-90 (2006).

16. Vetsch, E. et al. Optical interface created by laser-cooled atoms trapped in the evanescent field surrounding an optical nanofiber. Phys. Rev. Lett. 104, 203603 (2010).

17. Goban, A. et al. Demonstration of a state-insensitive, compensated nanofiber trap. Phys. Rev. Lett. 109, 033603 (2012).

18. Thompson, J. D. et al. Coupling a single trapped atom to a nanoscale optical cavity. Science 340, 1202-1205 (2013).

19. Goban, A. et al. Atom-light interactions in photonic crystals. Nature Commun. 5, 3808 (2014)

20. Kimble, H. J. The quantum internet. Nature 453, 1023-1030 (2008).

21. Greentree, A. D., Tahan, C., Cole, J. H. \& Hollenberg, L. C. L. Quantum phase transitions of light. Nature Phys. 2, 856-861 (2006).

22. Hartmann, M. J., Brandao, F. G. S. L. \& Plenio, M. B. Strongly interacting polaritons in coupled arrays of cavities. Nature Phys. 2, 849-855 (2006).

23. Angelakis, D. G., Santos, M. F. \& Bose, S. Photon-blockade-induced Mott transitions and $x y$ spin models in coupled cavity arrays. Phys. Rev. A 76, 031805 (2007).

24. Joannopoulos, J. D., Johnson, S. G., Winn, J. N. \& Meade, R. D. Photonic Crystals: Molding the Flow of Light 2nd edn (Princeton Univ. Press, 2008).

25. Kurizki, G. Two-atom resonant radiative coupling in photonic band structures. Phys. Rev. A 42, 2915-2924 (1990).

26. John, S. \& Wang, J. Quantum optics of localized light in a photonic band gap. Phys. Rev. B 43, 12772-12789 (1991).

27. John, S. \& Wang, J. Quantum electrodynamics near a photonic band gap: photon bound states and dressed atoms. Phys. Rev. Lett. 64, 2418-2421 (1990).

28. Bay, S., Lambropoulos, P. \& Mølmer, K. Atom-atom interaction in strongly modified reservoirs. Phys. Rev. A 55, 1485-1496 (1997).
29. Lambropoulos, P., Nikolopoulos, G. M., Nielsen, T. R. \& Bay, S. Fundamental quantum optics in structured reservoirs. Rep. Prog. Phys. 63, 455 (2000).

30. Shahmoon, E. \& Kurizki, G. Nonradiative interaction and entanglement between distant atoms. Phys. Rev. A 87, 033831 (2013).

31. González-Tudela, A., Hung, C.-L., Chang, D. E., Cirac, J. I. \& Kimble, H. J. Subwavelength vacuum lattices and atom-atom interactions in two-dimensional photonic crystals. Nature Photon. http://dx.doi.org/10.1038/nphoton.2015.54 (2015).

32. Yu, S.-P. et al. Nanowire photonic crystal waveguides for single-atom trapping and strong light-matter interactions. Appl. Phys. Lett. 104, 11103 (2014).

33. Agarwal, G., Gupta, S. \& Puri, R. Fundamentals of Cavity Quantum Electrodynamics (World Scientific Publishing, 1995).

34. Plenio, M. B., Huelga, S. F., Beige, A. \& Knight, P. L. Cavity-loss-induced generation of entangled atoms. Phys. Rev. A 59, 2468-2475 (1999).

35. Domokos, P. \& Ritsch, H. Collective cooling and self-organization of atoms in a cavity. Phys. Rev. Lett. 89, 253003 (2002).

36. Black, A. T., Chan, H. W. \& Vuletić, V. Observation of collective friction forces due to spatial self-organization of atoms: from Rayleigh to Bragg scattering. Phys. Rev. Lett. 91, 203001 (2003).

37. Baumann, K., Guerlin, C., Brennecke, F. \& Esslinger, T. Dicke quantum phase transition with a superfluid gas in an optical cavity. Nature 464, 1301-1306 (2010).

38. Hung, C.-L., Meenehan, S. M., Chang, D. E., Painter, O. \& Kimble, H. J. Trapped atoms in one-dimensional photonic crystals. New J. Phys. 15, 083026 (2013).

39. Markos, P. \& Soukoulis, C. M. Wave Propagation: From Electrons to Photonic Crystals and Left-Handed Materials (Princeton Univ Press, 2010).

40. Porras, D. \& Cirac, J. I. Effective quantum spin systems with trapped ions. Phys. Rev. Lett. 92, 207901 (2004).

41. Islam, R. et al. Emergence and frustration of magnetism with variable-range interactions in a quantum simulator. Science 340, 583-587 (2013).

42. Gross, M. \& Haroche, S. Superradiance: an essay on the theory of collective spontaneous emission. Phys. Rep. 93, 301-396 (1982).

43. Mattioli, M., Dalmonte, M., Lechner, W. \& Pupillo, G. Cluster Luttinger liquids of Rydberg-dressed atoms in optical lattices. Phys. Rev. Lett. 111, 165302 (2013).

44. Basko, D. M., Aleiner, I. L. \& Altshuler, B. L. On the problem of many-body localization. Preprint at http://arXiv.org/abs/cond-mat/0602510 (2006).

45. Longo, P., Schmitteckert, P. \& Busch, K. Few-photon transport in low-dimensional systems. Phys. Rev. A 83, 063828 (2011).

46. Firstenberg, O. et al. Attractive photons in a quantum nonlinear medium. Nature 502, 71-75 (2013).

47. Nayak, K. P. \& Hakuta, K. Photonic crystal formation on optical nanofibers using femtosecond laser ablation technique. Opt. Express 21, 2480-2490 (2013).

48. Chang, D. E., Jiang, L., Gorshkov, A. V. \& Kimble, H. J. Cavity QED with atomic mirrors. New J. Phys. 14, 063003 (2012).

49. Eichenfield, M., Chan, J., Camacho, R. M., Vahala, K. J. \& Painter, O. Optomechanical crystals. Nature 462, 78-82 (2009).

50. Dung, H. T., Knöll, L. \& Welsch, D.-G. Resonant dipole-dipole interaction in the presence of dispersing and absorbing surroundings. Phys. Rev. A 66, 063810 (2002).

\section{Acknowledgements}

The authors thank L. Tagliacozzo, P. Hauke, M. Lewenstein, A. González-Tudela, J.I. Cirac L. Jiang, J. Preskill, O. Painter, M. Lukin, J. Thompson and S. Gopalakrishnan for discussions. This work was supported by Fundacio Privada Cellex Barcelona, the MINECO Ramon y Cajal Program, the Marie Curie Career Integration Grant, the IQIM, an NSF Physics Frontiers Center, the DoD NSSEFF programme, DARPA ORCHID, AFOSR QuMPASS MURI, NSF PHY-1205729, NSF PFC at the JQI, NSF PIF, ARO, AFOSR, ARL and AFOSR MURI on Ultracold Polar Molecules.

\section{Author contributions}

J.S.D., H.H. and C.-L.H. performed the calculations. All authors contributed ideas. J.S.D. and D.E.C. wrote the manuscript.

\section{Additional information}

Supplementary information is available in the online version of the paper. Reprints and permissions information is available online at www.nature.com/reprints. Correspondence and requests for materials should be addressed to J.S.D.

\section{Competing financial interests}

The authors declare no competing financial interests. 